\title{
Chromosome integrity checkpoints in stem and progenitor cells: transitions upon differentiation, pathogenesis, and aging
}

\author{
Andreas Brown ${ }^{1}$ (D) Hartmut Geiger ${ }^{1,2}$ \\ Received: 19 January 2018 / Revised: 22 July 2018 / Accepted: 25 July 2018 / Published online: 31 July 2018 \\ (c) The Author(s) 2018
}

\begin{abstract}
Loss of chromosome integrity is a major contributor to cancer. Checkpoints within the cell division cycle that facilitate the accuracy and outcome of chromosome segregation are thus critical pathways for preserving chromosome integrity and preventing chromosomal instability. The spindle assembly checkpoint, the decatenation checkpoint and the post-mitotic tetraploidy checkpoint ensure the appropriate establishment of the spindle apparatus, block mitotic entry upon entanglement of chromosomes or prevent further progression of post-mitotic cells that display massive spindle defects. Most of our knowledge on these mechanisms originates from studies conducted in yeast, cancer cell lines and differentiated cells. Considering that in many instances cancer derives from transformed stem and progenitor cells, our knowledge on these checkpoints in these cells just started to emerge. With this review, we provide a general overview of the current knowledge of these checkpoints in embryonic as well as in adult stem and progenitor cells with a focus on the hematopoietic system and outline common mis-regulations of their function associated with cancer and leukemia. Most cancers are aging-associated diseases. We will thus also discuss changes in the function and outcome of these checkpoints upon aging of stem and progenitor cells.
\end{abstract}

Keywords Mitotic checkpoints $\cdot$ Spindle assembly checkpoint $\cdot$ Decatenation checkpoint $\cdot$ Chromosomal instability $\cdot$ Stem and progenitor cells $\cdot$ Leukemia $\cdot$ HSCs $\cdot$ HSPCs

\section{Principles of mitotic checkpoint signaling}

One major feature of hematopoietic neoplasms is chromosomal instability (CIN). In $95 \%$ of all cases bone marrow (BM) cells of chronic myeloid leukemia (CML) patients, for instance, are present with the Philadelphia chromosome arising from a translocation between chromosomes 9 and 22 [1]. Moreover, cells from most acute myeloid leukemia (AML) subsets contain cytogenetic aberrations, such as $t(8 ; 21)$ or $t(15 ; 17)$ [2]. These findings emphasize the importance of cellular mechanisms that limit CIN to restrict

Hartmut Geiger

hartmut.geiger@uni-ulm.de

Andreas Brown

andreas.brown@uni-ulm.de

1 Institute of Molecular Medicine, Ulm University, Life Science Building N27, James Franck-Ring/Meyerhofstrasse, 89081 Ulm, Germany

2 Division of Experimental Hematology and Cancer Biology, Cincinnati Children's Hospital Medical Center, 3333 Burnet Ave, Cincinnati, OH 45229, USA disease initiation. Therefore, a great variety of crucial mechanisms supporting the process of sister chromatid separation and chromosome stability is active before, during, or after mitotic progression of the cell division cycle, such as the spindle assembly checkpoint (SAC) and the decatenation checkpoint (DC). Whereas the SAC is only active in (pro) metaphase and disabled shortly before anaphase onset in the course of mitotic or meiotic progression, the DC is triggered during the late phase of $\mathrm{G} 2$ of the cell cycle. Together, both mechanisms prevent chromosomal anomalies, such as translocations, trisomies, and insertions/deletions, hence ensuring genomic integrity of daughter cells upon cell division. In addition, other pathways such as the G1 tetraploidy checkpoint, sometimes referred to as post-mitotic checkpoint, or signal cascades that facilitate precise centrosome duplication have been described: these mechanisms have been considered to contribute to chromosomal integrity as well, although their exact implications in mammalian cells have been discussed $[3,4]$.

Here, we focus on the current knowledge of the mechanisms of these late G2, mitotic and post-mitotic checkpoints in stem and progenitor cells and highlight differences in 
comparison with cell lines and differentiated cells. We also present the current understanding of how these checkpoints are altered in diseases and upon aging. We omit other checkpoint mechanisms of genome integrity, such as DNA damage response pathways, which are already discussed elsewhere in detail [5-7]. Figure 1 lists various known mitotic checkpoint alterations and their involvement in hematopoietic malignancies and other types of cancers. They will be discussed in the subsequent paragraphs.

\section{The SAC ensures fidelity of chromosome segregation in stem and progenitor cells}

The SAC is critical for temporarily arresting mitotic progression to enable the accurate coordination between kinetochores and the spindle apparatus or to resolve erroneous chromosomal attachments: sister chromatids must not be separated until all kinetochores are completely and tightly aligned at the metaphase plate. Only in this scenario, the SAC is switched off to finally trigger sister chromatid segregation. If the spindle defects are too severe, though, apoptosis is initiated by p53-dependent and -independent pathways $[8,9]$. The SAC involves the inhibitory activity of the mitotic checkpoint complex (MCC) which includes, among others, Cell division cycle 20 (Cdc20), one of the main coactivators of the anaphase promoting complex (APC/C). In response to the recognition of imprecise or false microtubule-kinetochore attachments, several kinetochore components are phosphorylated by Monopolar spindle protein 1 (Mps1) and Aurora B, among others, and MCC component Mitotic arrest deficient 2 (Mad2) rapidly adjusts its native conformation $[10,11]$. This switch provokes a signaling cascade that is distributed throughout the nucleus, forcing Cdc20 to incorporate within the MCC. Upon satisfaction of the SAC, Cdc20 is released-now able to stimulate the APC/C [12]. This multi-subunit E3-ubiquitin ligase targets securin (Pttg1) and cyclinB1, the two mayor inhibitors of separase for their proteasomal degradation [13]: being unaffected by its negative regulators, separase catalyzes the sitespecific proteolysis of the centromeric cohesion component Scc1 $(\operatorname{Rad} 21)$ : anaphase onset is initiated. This whole process requires tight regulation. If severe DNA aberrations or unequal chromosome numbers remain undiscovered after metaphase, they will be distributed to daughter cells and ultimately contribute to cancerogenesis [14]. The activity of the SAC and the expression of associated genes have been demonstrated in many tissues of the human body - from fast growing mammary epithelial cells to germ cells undergoing meiotic division $[15,16]$. Thus, the molecular mechanisms of the SAC have been extensively studied, however, mainly in yeast or cancer cell lines, such as HCT116 and Hela. Here, often unnatural karyotypes, aneuploidies or large chromosome numbers are present, making a functional SAC inevitable, even for these highly transformed cells [17]. However, information on the SAC in untransformed somatic tissues and particularly in (adult) stem cells has lagging behind, with novel and exciting data though emerging over the last couple of years.

In one of the first studies regarding this topic, Rohrabaugh and co-workers demonstrated that hematopoietic stem and progenitor cells (HSPCs) arrest at the G2/M boundary in response to treatment with the spindle drug nocodazole which blocks tubulin polymerization and induce cell death
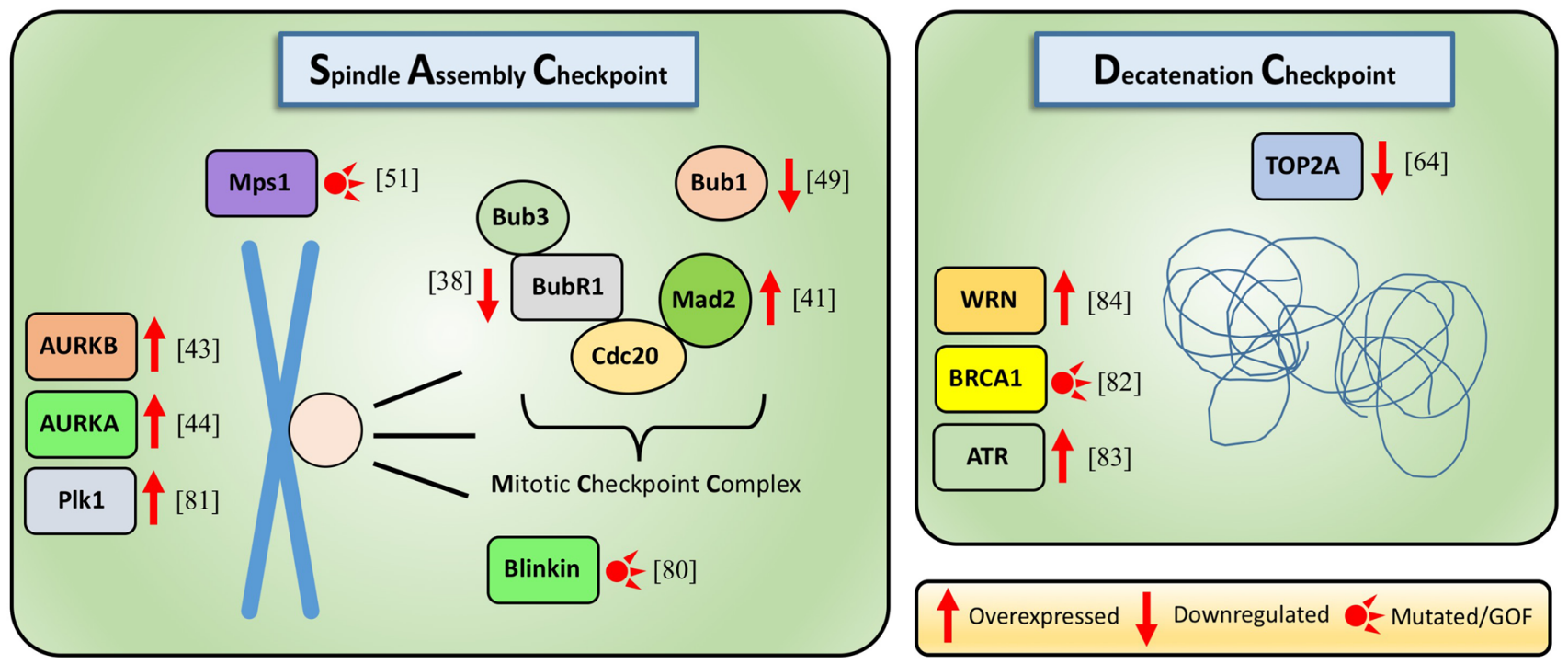

Fig. 1 Genes involved in the spindle assembly checkpoint or decatenation checkpoint activity and their deregulation in hematological malignancies 
[18]. Interestingly, in murine embryonic stem (ES) cells SAC activity was demonstrated, though apoptosis was not induced in response to prolonged exposure to nocodazole which activated the checkpoint. This behavior might allow for a high tolerance to chromosomal abnormalities and polyploidy, according to the authors, and may at least in part account for the appearance of trisomic disorders [19]. We recently confirmed the presence of the SAC in HSPCs [20] and demonstrated that it is at least in part required for proper engraftment of progenitors in peripheral blood and hematopoietic colony formation. Similar to differentiated cells but in contrast to murine ES cells, prolonged treatment with anti-mitotic drugs, such as nocodazole or taxol to activate the checkpoint causes p53-dependent apoptosis. Analogous to other types of cells, the SAC depends on the activity of Mps1 and Aurora B since chemical inhibition of these components caused a checkpoint override as assessed by cell cycle analysis. Strikingly, in hematopoietic stem cells (HSCs), inhibition of the SAC, while present, had only mild effects, as chemical inhibition with the Mps 1 inhibitor reversine neither increased the number of chromosomal aberrations nor did it negatively influence late engraftment after competitive transplantation (a feature, which has been shown to be mainly driven by HSCs [21]). In contrast, the SAC was shown to be of critical importance for more committed hematopoietic progenitors, such as Lin-cKit+ cells. Here, checkpoint inhibition induced a massive failure to form colonies on methylcellulose and negatively influenced their engraftment potential [20]. Interestingly, in murine HSPCs the SAC only in part depends on the MCC component Bub1related kinase (BubR1), as BubRl-haploinsufficiency did not alter cell cycle dynamics and the clonal colony formation ability of HSPCs [20]. Another study revealed that HSCs hypomorphic for BubRl $\left(\mathrm{Bub}_{\mathrm{b}} \mathrm{b}^{\mathrm{H} / \mathrm{H}}\right)$ lose their engraftment potential upon secondary transplantation in recipient mice and after tertiary transplantation $\mathrm{Bub} 1 \mathrm{~b}^{\mathrm{H} / \mathrm{H}}$ donor-derived HSCs completely failed to engraft. These findings unveil that, ultimately, BubRl deficiency indeed causes premature HSC exhaustion, but only after several rounds of cell division, consistent with the reported minor role of that gene for the SAC in HSCs [22]. Interestingly, upon overexpression of BubR1 in mice, aneuploidy and the incidence of agerelated cancers were decreased, whereas overall lifespan was increased [23, 24]. Consequently, BubR1 may also account as a lifespan gene [25]. Another study addressed SAC function in human hematopoietic progenitors: here, the authors conducted experiments in BM cells from Mad2 haploinsufficient $\left(\mathrm{Mad}^{+/-}\right)$mice and examined absolute numbers as well as cell cycle dynamics of various hematopoietic subpopulations. Although mature progenitors, such as granulocyte precursors exhibited a normal behavior in terms of cycling activity and absolute cell numbers, large alterations in absolute cell numbers, high apoptosis rates and enhanced proliferation upon cytokine stimulation of immature progenitors were observed. Interestingly, the authors revealed that in human hematopoietic progenitors SAC component Mad2 associates with c-Kit, an important receptor tyrosine kinase of HSPCs [26]. Similar observations with respect to Mad2 were reported for skin cells: upon depletion of Mad2, the hair follicle bulge stem cell pool was diminished, while there were no large differences in term of function and numbers of interfollicular epidermal cells. However, these mice lost most of their hair after birth, and furthermore, chromosomal analysis of the epidermis revealed high amounts of aneuploidy, a known consequence of SAC deficiency [27]. The study further supported the concept that an impaired SAC has different outcomes on various cells of the same tissue. Last, the activity of Mad2 has been proposed to also affect asymmetric/symmetric cell division of stem cells by influencing their spindle positions during mitosis. Hence, SAC component Mad2 might be a novel factor involved in controlling stem cell differentiation and self-renewal [26, 28]. A major result of abrogated SAC activity is the occurrence of trisomic disorders. Indeed, it is assumed that trisomic cells present with an inherent defect in the SAC. Pfau et al. described that fetal HSCs isolated from mice carrying constitutional trisomies of chromosome 11 or 16 displayed severe engraftment defects [22]. The activity of the SAC could also be demonstrated in muscle progenitor and stem cells (satellite cells): upon tamoxifen-mediated knockdown of Mps1, these cells failed to differentiate and were unable to expand [29]. In murine megakaryocytes, which are polyploid and have chromosomes numbers of up to $256 \mathrm{~N}$, the SAC has a crucial impact on megakaryopoiesis in terms of number of splenic megakaryocytes while checkpoint failure triggered by BubRl knockout did not result in a significant lack of thrombocytes [30].

The SAC has been demonstrated to be present in stem cells from other distinct organisms as well: in Drosophila embryos downregulation of the SAC by deletion of Mad2 caused massive mitotic failure and depletion of the neuronal progenitor cell pool as well as medulla reduction and enlarged central brain sizes [31]. In malignant fly tumor neural stem cells, deregulation of the SAC by genetic knockdown provoked impairment of sister chromatid segregation and aneuploidy. Crucially, these stem cells were not able to form colonies anymore. Disruption of SAC component Aurora A, however, did not lead to inhibition of aneuploidy although the SAC was inhibited [32,33]. Last, in C. elegans, SAC inhibition by Mad2 knockdown in germline stem and progenitor cells triggers a corrupted spindle assembly in terms of spindle length and a delay in mitotic progression. Interestingly, whereas $M a d 2, B u b R I$ and $B u b 3$ are reported to be conserved between $C$. elegans and higher organisms, a homolog for the checkpoint kinase Mps1 was not found in this nematode [34]. Together, these findings support the 
conclusion that the SAC is of general importance for stem and progenitor cells in most organisms. In higher organisms, especially in mammals, the pool of data suggests that the lack of correct activation of the SAC has varying outcomes, depending on the tissue and the affected SAC-related genes.

\section{Changes in SAC signaling in cancer}

Chromosomal instability provokes both tumor initiation and progression, especially in hematopoietic neoplasms. Usually, the underlying cells driving leukemias such as acute myelogenous leukemia (AML) or CML are malignantly transformed HSPCs [35-37]. Strikingly, many studies could demonstrate the abrogation or malfunction of the SAC in leukemia cells taken from patients. For instance, it has been shown that in most BM samples from AML patients, the important SAC regulator $B u b R l$ is downregulated, whereas other SAC-involved genes such as budding uninhibited by benzimidazoles $1(B u b l)$ and $B u b 3$ were not mis-regulated [38]. The reduced expression of BubRl goes along with a strong deregulation of the SAC in these cells: The two main regulators of separase, cyclinB 1 and securin are prematurely degraded and high levels of chromosomal aberrations, such as trisomies were observed. However, in response to overexpression of BubRl, SAC activity and sensitivity to nocodazole can be regained. Previously SAC-deficient cells stabilize their cyclin B1 amounts after treatment with anti-mitotic drugs and the frequency of chromosome missegregation is decreased, whereas apoptosis levels are elevated [38]. Another SAC component, Mad2, which is required for instantly distributing the SAC signal throughout the nucleus, has been described to be mis-regulated in leukemia as well [39, 40]. Indeed, overexpression of Mad2 in transgenic mice promoted aneuploidy, anaphase bridges, chromosome breaks as well as initiation of tumors, such as lymphoma and hepatocellular carcinoma. Interestingly, elevated levels of Mad2 did not appear to have an impact on further tumor progression, suggesting that Mad2 misregulation is primarily involved in initial steps of tumor formation [41]. Moreover, in leukemic (AML) cells positive for the fusion gene AML-ETO (AEtr), it was demonstrated that the SAC was deregulated since these cells failed to arrest in response to anti-mitotic drugs. In such cells, BubRl levels were reduced upon nocodazole treatment, causing mis-regulated APC/C activity and hence premature securin degradation. Interestingly, other checkpoint proteins, such as Mad2 or Bub3 were not downregulated, indicating a specific correlation of the presence of AEtr and decreased BubR1 expression [42]. Further on, expression levels of the SAC components Aurora A/B have been reported for AML cells [43], whereas upregulation of Aurora A has also been connected to the initiation of myelodysplastic syndrome (MDS)
[44]. This heterogeneous class of various blood cancers also in most cases emerges from transformed HSPCs [45, 46]. The van Deursen laboratory showed that overexpression of the $\mathrm{MCC}$ regulator $\mathrm{Bubl}$ in mice induced tumor formation [47], while the level of expression of the gene was reduced in AML specimens and cell lines, such as K562 and HL60 $[48,49]$. Other analyses revealed high expression of $\operatorname{Mad} 2$, Aurora B and Cdc20 in MDS. Interestingly, each MDS subtype was reported to have its own expression profile of SAC genes. High expression, especially of Mad2 and $\mathrm{Cdc} 20$ was associated with thrombocytopenia and an overall poor survival rate [45]. That implies that the level of impairment of the SAC triggered by $\mathrm{Cdc} 20$ and Mad2 mis-regulation might directly contribute to the severity of the disease. In Runt-related transcription factor 1 (RunxX1)-mutated acute lymphoblastic leukemia (ALL) cells, severe SAC malfunctions were also described. With the SAC regulator Mad2 being downregulated, these cells failed to sufficiently arrest upon anti-mitotic drug treatment and ALL-derived cells displayed high amounts of trisomies and other mayor chromosomal abnormalities [50]. Mutations in the important MCC regulator Mps1 have been implied in the initiation of ALL and AML as well, and indeed, inhibitors targeting Mps1 are already in clinical trials [51, 52]. Finally, the checkpoint has also been studied in transformed induced pluripotent stem (iPS) cells that induced aggressive teratomas [53]: these teratoma cells displayed aberrant cell cycle regulation and CIN, which could be linked to high expression of the checkpoint kinase Aurora A. Indeed, co-inhibition of Aurora A during iPS generation prevented the transformation process [54]. These reports demonstrate that transformed stem cells can also exhibit high levels of expression of genes important for SAC function that contribute to pathology.

In summary, a broad range of hematological malignancies, such as MDS, AML and ALL, have been reported to be present with failed or mis-regulated SAC activity, primarily due to mis-expression (both higher and lower than normal) of genes related to SAC activity. The data imply that chromosomal aberrations such as CIN and cell cycle deregulation in cancer might be a direct consequence of SAC malfunction in stem and progenitor cells, since these cells are very often the driver of these diseases.

\section{DC and tetraploidy checkpoint mechanisms and their relevance in stem cells and cancer}

The decatenation checkpoint (DC) is already active in the late $\mathrm{G} 2$ phase, the third period of interphase, and thus earlier than the SAC. In this phase of the cell cycle, sister chromatids, though still being decondensed, must already be physically connected by cohesin ring complexes, whereas the chromatin of complementary strands must not be 
intertwined. Upon entanglement, further cellular progression is blocked, enabling the physical separation of these DNA strands by the activity of topoisomerase II $\alpha$ (TOP2A) [55-57]. The DC is not directly related to the G2/M checkpoint that is exclusively activated in response to DNA damage; however, both mechanisms share a distinct set of signaling proteins, such as Ataxia telangiectasia and Rad3related protein (ATR) and Polo-like kinase 1 (Plk1) [58]. It has been claimed that the DC is absent in murine ES and neural progenitor cells as well as human hematopoietic progenitor $(\mathrm{CD} 34+)$ cells as stem cell multipotency may not be compatible with proper DC activity. Upon differentiation of ES cells into more committed progenitors, interestingly, the checkpoint regained activity [59]. Inversely, whereas the activity of this checkpoint was demonstrated in a broad range of cell lines and differentiated tissues, it was absent in various carcinoma and lung cancer cell lines, such as A549 and ACC-LC-172 [60-62]. Therefore, it is discussed that the absence of the DC may be a driver of cancer progression [63], or, it might be an indicator of the stem cell-like character of the cells underlying the cancer. Indeed, also AML cells do not activate the DC in response to entanglement of chromosomes. Moreover, AML cells show constitutive high expression of the protein Metnase, which is able to support TOP2A function by its histone methylation activity forcing chromosome decatenation without the cell having to arrest [64]. Not surprisingly, even in the presence of TOP2A inhibitors AML cells are able to promote decatenation without an arrest at G2, while reduction of Metnase levels can re-activate DC activity in AML cell lines [64, 65]. DC deficiency has been also reported for most melanoma cell lines. However, in these cells inhibition of TOP2A cannot be compensated by Metnase, as in AML cells, provoking cell cycle arrest and apoptosis [66]. Similarly, colon cancer cells, such as HCT116 and HT-29, which have a defective G2 decatenation checkpoint as well, can be eliminated by TOP2A inhibitors. [67].

In conclusion, the data published imply that DC activity is especially important for differentiated cells and that DC deficiency might be a general feature of cancer cells, whereas no evidence exists so far with respect to its activity in primitive and undifferentiated cells. Its absence may thus be especially involved in tumor progression. However, additional research is mandatory to address the role of the DC especially in stem and progenitor cells.

The post-mitotic checkpoint, also termed G1 tetraploidy checkpoint, was reported to arrest cells which suffered from severe spindle defects resulting from premature mitotic exit or SAC failure p53-dependently finally in early G1 [68]. This pathway is, therefore, deregulated in cancer cells with reduced or absent p53 activity [55]. Some researchers claim that also mature and untransformed mammalian cells do not activate this checkpoint $[3,4]$. Research from our laboratory suggested the presence of this checkpoint at least in HSPCs. Upon inhibition of cycling of HSPCs with the specific Mps1 inhibitor reversine, we observed a strong and durable G1 arrest. Furthermore, reversine-treated progenitor cells were not able to form colonies on methylcellulose anymore, implying a permanent cell cycle arrest. This finding demonstrated the presences of a post-mitotic checkpoint blocking HSPCs with corrupted SAC activity from further cycling [20]. Similarly, the group of Andrew Brack described the existence of a p21 ${ }^{\mathrm{Cip} 1}(\mathrm{Cdkn} 1 \mathrm{a})$-dependent post-mitotic checkpoint in muscle stem and progenitor cells, also activated by Mps1 inhibition [29]. According to the authors, this mechanism may be involved in preventing early malignant transformation of stem and progenitor cells.

Finally, a small number of reports already addressed a particular checkpoint arresting cells at the G1/S boundary in response to loss of centrosome integrity which includes improper centrosome numbers, for instance. Key players of this checkpoint are p53 and p21, analogous to the G1/S DNA damage response [69]. Whether this mechanism is active in stem and progenitor cells and whether it has an impact during cancer initiation or progression has not yet been studied, though.

\section{Changes of mitotic checkpoint signaling during the aging process}

Cancer is an aging-associated disease [70]. In general, our knowledge on aging-associated changes in the function of mitotic checkpoints is very limited. One paradigm claims that an increase in the number of DNA mutations upon aging is linked to the higher incidence of cancer in the elderly [71]. However, while the number of single point mutations found in BM samples from AML patients increases linearly with age, the number of AML incidents raises exponentially during the same time frame [72]. This discrepancy implies that deregulation of regulatory pathways, such the SAC, might in theory also play a role in cancer initiation. One report, however, suggested that there is no correlation between SAC activity and longevity and that the reliability and function of the SAC decline rather with the number of mitotic divisions and not primarily with age [73]. Human cells probably maintain a very vigorous SAC upon aging since a direct correlation between checkpoint robustness and body mass was described [74]. Further findings suggest that aneuploidy in aged mouse eggs is not primarily caused by a defective SAC, in contrast to studies from other research groups $[18,75]$. Moreover, the group of Andrew Brack reported that although the SAC is essential for maintaining muscle stem cell function, aging does not have an impact on the robustness of the SAC itself [29]. Inversely, there is a correlation between age and expression of the checkpoint genes Mad2, Aurora 
$\mathrm{B}$ and Cdc20 in myelodysplastic syndrome (MDS), an characteristic aging-associated dysplasia of the blood-forming system. The same study also demonstrated a correlation of expression levels of these genes and the developmental stage of MDS, as already discussed in the previous paragraph [45].

Mechanistically, expression of BubRl has been reported to continuously decline upon aging in several tissues such as ovary and testis, whereas its expression in other tissues or transcription of different checkpoint genes such as Bub3 were reported to be unaltered [76]. High expression of the SAC component BubR1 has been linked to longevity as well as to low rates of chromosomal aberrations and aneuploidy. Upon overexpression of $B u b R l$ in vivo, direct consequences of low SAC activity, such as false microtubule-kinetochore attachments, could be avoided [25].

In summary, the relative robustness of the SAC indicates that checkpoint deregulation may not be an early event during carcinogenesis but might rather be involved in tumor progression (as suggested in Fig. 2). Whether there is a role in the elevated initiation of cancer in the elderly needs to be further investigated, as the published data do not allow for unequivocal conclusions on: (a) the extent to which aging affects mitotic checkpoints and (b) whether SAC alterations contribute to the increase of cancer in the elderly. Other checkpoints, such as DNA damage response pathways, indeed have been reported to change upon aging [77], and might thus be also involved in the contribution to agingassociated cancers.

\section{Directions}

Our knowledge on mitotic checkpoint signaling in stem and progenitor cells is still limited but over the recent years novel findings have started to shed light on the mechanisms that govern it and its importance for health and disease. Studies that investigate these checkpoints in HSPCs, muscle satellite cells, neuronal and ES cells have been providing initial insights in their function. This is especially relevant since many reports demonstrate the abrogation of the SAC or the DC, especially in hematopoietic neoplasms, such as MDS, CML and AML but also other types of cancers, such as lung cancer. In these cases, cancer most likely emerges from transformed stem and progenitor cells. Here, very often chromosomal disorders, such as translocations or aneuploidies, are foundone major consequence when mitotic checkpoints fail to operate reliably. These results emphasize the importance of investigations of mitotic checkpoint signaling in still untransformed stem and progenitor cells and the contribution of failing checkpoints to cancer initiation and progression, which might allow to therapeutically target such checkpoints. Indeed, it was demonstrated that re-activation of the SAC in AML cells promotes sensitivity to chemotherapeutics such as taxol [38]. In other cases, inhibition of Aurora kinases which were found to be overexpressed in cancer cells promoted apoptosis [78, 79]. Finally, the

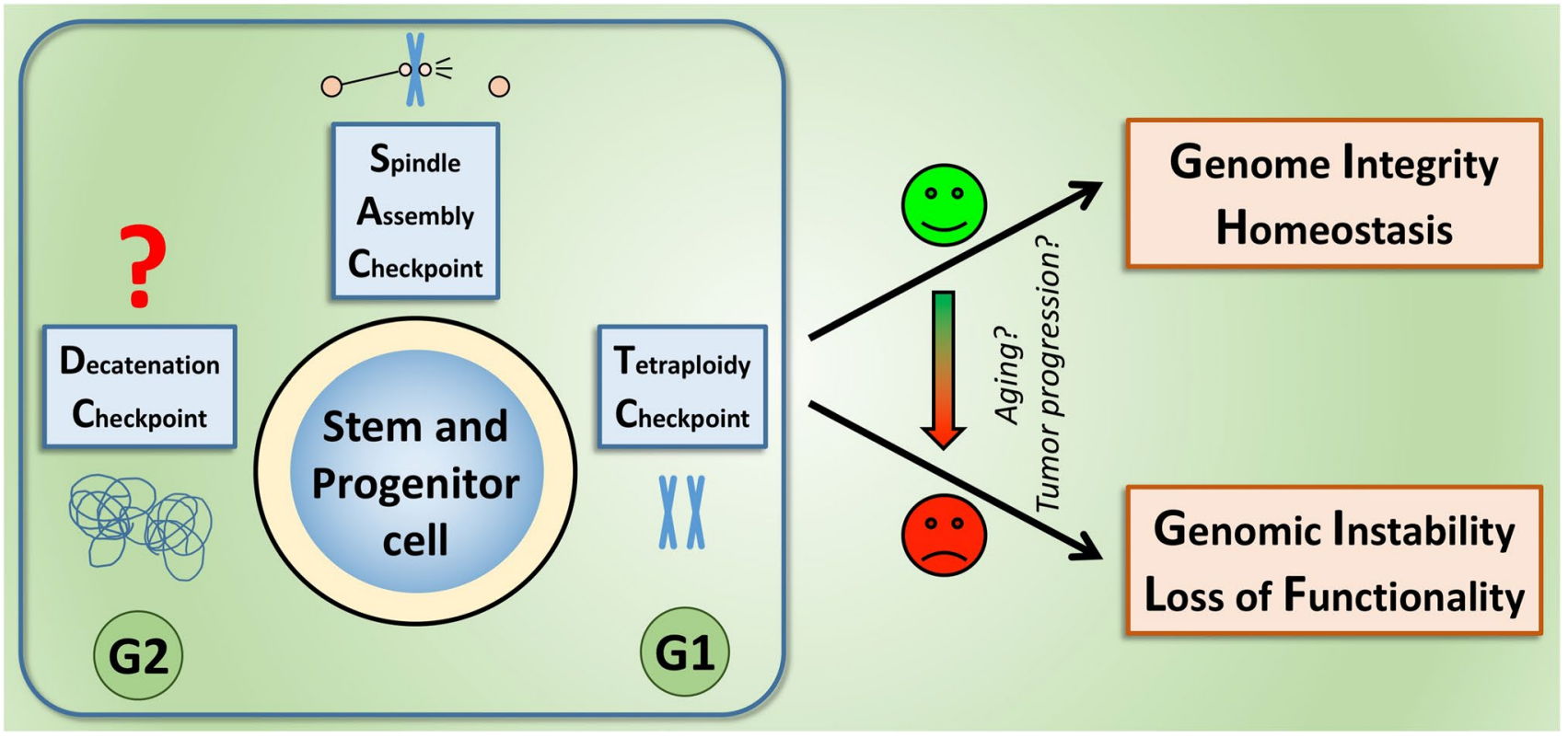

Fig. 2 Mechanisms that ensure the fidelity of chromosome segregation, especially the SAC are required to maintain genome integrity and homeostasis of stem and progenitor cells. Whereas a growing number of studies demonstrate the activity of the SAC and the tetraploidy (post-mitotic) checkpoint in these cells, it is not clear yet, whether they also activate a pathway homologous to the DC. Strikingly, many reports illustrate that without these mechanisms, stem and progenitor cells may take the route towards chromosomal instability, provoking loss of functionality, transformation and, ultimately, cancer 
re-activation of the DC in AML-derived cell lines was able to induce cell cycle arrest and apoptosis. Consequently, further research into the function of these checkpoints in both young and aged stem and progenitor cells may allow the development of novel pharmaceutical concepts for the treatment of cancer.

Funding This work was supported by grants from the Deutsche Forschungsgemeinschaft (SFB1074) to H.G. and a "Bausteinprogramm" to A.B. from the Medical Faculty of Ulm University. Work in the Geiger laboratory is in addition supported by NIH Grants AG040118 and DK104814 and the DFG GRK 2254 HEIST and 1789 CEMMA and SFBs 1149 and 1275 and the BMBF (SyStaR).

\section{Compliance with ethical standards}

Conflict of interest The authors do not have any conflict of interest.

Open Access This article is distributed under the terms of the Creative Commons Attribution 4.0 International License (http://creativeco mmons.org/licenses/by/4.0/), which permits unrestricted use, distribution, and reproduction in any medium, provided you give appropriate credit to the original author(s) and the source, provide a link to the Creative Commons license, and indicate if changes were made.

\section{References}

1. Cuneo A, Ferrant A, Michaux JL et al (1996) Philadelphia chromosome-positive acute myeloid leukemia: cytoimmunologic and cytogenetic features. Haematologica 81:423-427

2. Nishii K, Usui E, Katayama $\mathrm{N}$ et al (2003) Characteristics of $\mathrm{t}(8 ; 21)$ acute myeloid leukemia (AML) with additional chromosomal abnormality: concomitant trisomy 4 may constitute a distinctive subtype of $\mathrm{t}(8 ; 21)$ AML. Leukemia 17:731-737. https:// doi.org/10.1038/sj.leu.2402871

3. Uetake Y, Sluder G (2004) Cell cycle progression after cleavage failure: Mammalian somatic cells do not possess a "tetraploidy checkpoint". J Cell Biol 165:609-615. https://doi.org/10.1083/ jcb. 200403014

4. Wong C, Stearns T (2005) Mammalian cells lack checkpoints for tetraploidy, aberrant centrosome number, and cytokinesis failure. BMC Cell Biol 6:6. https://doi.org/10.1186/1471-2121-6-6

5. Moehrle BM, Geiger H (2016) Aging of hematopoietic stem cells: DNA damage and mutations? Exp Hematol 44:895-901. https:// doi.org/10.1016/j.exphem.2016.06.253

6. Schnerch D, Yalcintepe J, Schmidts A et al (2012) Cell cycle control in acute myeloid leukemia. Am J Cancer Res 2:508-528

7. Sperka T, Wang J, Rudolph KL (2012) DNA damage checkpoints in stem cells, ageing and cancer. Nat Rev Mol Cell Biol 13:579590. https://doi.org/10.1038/nrm3420

8. Orth JD, Loewer A, Lahav G, Mitchison TJ (2012) Prolonged mitotic arrest triggers partial activation of apoptosis, resulting in DNA damage and p53 induction. Mol Biol Cell. https://doi. org/10.1091/mbc.E11-09-0781

9. Beeharry N, Yen TJ (2009) p53-dependent apoptosis in response to spindle damage is linked to loss of Bub1. Cancer Biol, Ther

10. Funabiki H, Wynne DJ (2013) Making an effective switch at the kinetochore by phosphorylation and dephosphorylation. Chromosoma 122:135-158
11. Musacchio A (2015) Closing the Mad2 cycle. Elife 4:1-3. https ://doi.org/10.7554/eLife.08283

12. Izawa D, Pines J (2011) How APC/C-Cdc20 changes its substrate specificity in mitosis. Nat Cell Biol 13:223-233. https:// doi.org/10.1038/ncb2165

13. Hagting A, Den Elzen N, Vodermaier HC et al (2002) Human securin proteolysis is controlled by the spindle checkpoint and reveals when the APC/C switches from activation by $\mathrm{Cdc} 20$ to Cdh1. J Cell Biol 157:1125-1137. https://doi.org/10.1083/ jcb.200111001

14. Musacchio A, Salmon ED (2007) The spindle-assembly checkpoint in space and time. Nat Rev Mol Cell Biol 8:379-393. https ://doi.org/10.1038/nrm2163

15. Privette LM, Weier JF, Nguyen HN et al (2008) Loss of CHFR in human mammary epithelial cells causes genomic instability by disrupting the mitotic spindle assembly checkpoint. Neoplasia 10:643-652. https://doi.org/10.1593/neo.08176

16. Gorbsky GJ (2015) The spindle checkpoint and chromosome segregation in meiosis. FEBS J 282:2471-2487

17. Tighe A, Johnson VL, Albertella M, Taylor SS (2001) Aneuploid colon cancer cells have a robust spindle checkpoint. EMBO Rep 2:609-614. https://doi.org/10.1093/embo-reports/kve127

18. Rohrabaugh S, Mantel C, Broxmeyer HE (2008) Mouse hematopoietic stem cells, unlike human and mouse embryonic stem cells, exhibit checkpoint-apoptosis coupling. Stem Cells Dev 17:1017-1020. https://doi.org/10.1089/scd.2007.0260

19. Mantel C, Guo Y, Lee MR et al (2007) Checkpoint-apoptosis uncoupling in human and mouse embryonic stem cells: a source of karyotpic instability. Blood 109:4518-4528. https://doi. org/10.1182/blood-2006-10-054247

20. Brown A, Pospiech J, Eiwen K et al (2017) The spindle assembly checkpoint is required for hematopoietic progenitor cell engraftment. Stem Cell Rep 9:1-10. https://doi.org/10.1016/j.stemc r.2017.09.017

21. Busch K, Klapproth K, Barile M et al (2015) Fundamental properties of unperturbed haematopoiesis from stem cells in vivo. Nature 518:542-546. https://doi.org/10.1038/nature14242

22. Pfau SJ, Silberman RE, Knouse KA, Amon A (2016) Aneuploidy impairs hematopoietic stem cell fitness and is selected against in regenerating tissues in vivo. Genes Dev 30:1395-1408. https:// doi.org/10.1101/gad.278820.116

23. Matsumoto T, Baker DJ, D'Uscio LV et al (2007) Aging-associated vascular phenotype in mutant mice with low levels of BubR1. Stroke 38:1050-1056. https://doi.org/10.1161/01.STR.00002 57967.86132 .01

24. Hartman TK, Wengenack TM, Poduslo JF, van Deursen JM (2007) Mutant mice with small amounts of BubR1 display accelerated age-related gliosis. Neurobiol Aging 28:921-927. https://doi. org/10.1016/j.neurobiolaging.2006.05.012

25. Baker DJ, Dawlaty MM, Wijshake T et al (2013) Increased expression of BubR1 protects against aneuploidy and cancer and extends healthy lifespan. Nat Cell Biol 15:96-102. https://doi.org/10.1038/ ncb2643

26. Ito S, Mantel CR, Han MK et al (2007) Mad2 is required for optimal hematopoiesis: Mad2 associates with c-Kit in MO7e cells. Blood 109:1923-1930. https://doi.org/10.1182/blood-2006-06030841

27. Foijer F, DiTommaso T, Donati G et al (2013) Spindle checkpoint deficiency is tolerated by murine epidermal cells but not hair follicle stem cells. Proc Natl Acad Sci USA 110:2928-2933. https:// doi.org/10.1073/pnas.1217388110

28. Mantel C, Broxmeyer HE (2007) A new connection between the spindle checkpoint, asymmetric cell division and cytokine signaling. Cell Cycle 6:144-146

29. Kollu S, Abou-Khalil R, Shen C, Brack AS (2015) The spindle assembly checkpoint safeguards genomic integrity of skeletal 
muscle satellite cells. Stem Cell Rep 4:1061-1074. https://doi. org/10.1016/j.stemcr.2015.04.006

30. Wang Q, Liu T, Fang Y et al (2004) BUBR1 deficiency results in abnormal megakaryopoiesis. Blood 103:1278-1285. https://doi. org/10.1182/blood-2003-06-2158

31. Poulton JS, Cuningham JC, Peifer M (2017) Centrosome and spindle assembly checkpoint loss leads to neural apoptosis and reduced brain size. https://doi.org/10.1083/jcb.201607022

32. Caous R, Richard-Parpaillon L, Giet R (2016) Drosophila Aurora A regulates mitotic timing in cancer stem cells: possible therapeutic implications. Mol Cell Oncol 3:e1140261. https:// doi.org/10.1080/23723556.2016.1140261

33. Caous R, Pascal A, Romé P et al (2015) Spindle assembly checkpoint inactivation fails to suppress neuroblast tumour formation in aurA mutant Drosophila. Nat Commun. https:// doi.org/10.1038/ncomms9879

34. Gerhold AR, Ryan J, Vallée-Trudeau JN et al (2015) Investigating the regulation of stem and progenitor cell mitotic progression by in situ imaging. Curr Biol 25:1123-1134. https://doi. org/10.1016/j.cub.2015.02.054

35. Bonnet D, Dick JE (1997) Human acute myeloid leukemia is organized as a hierarchy that originates from a primitive hematopoietic cell. Nat Med 3:730-737

36. Goardon N, Marchi E, Atzberger A et al (2011) Coexistence of LMPP-like and GMP-like leukemia stem cells in acute myeloid leukemia. Cancer Cell 19:138-152. https://doi.org/10.1016/j. ccr.2010.12.012

37. Shet AS, Jahagirdar BN, Verfaillie CM (2002) Chronic myelogenous leukemia: mechanisms underlying disease progression. Leukemia 16(8):1402-1411

38. Schnerch D, Schmidts A, Follo M et al (2013) BubR1 is frequently repressed in acute myeloid leukemia and its re-expression sensitizes cells to antimitotic therapy. Haematologica 98:1886-1895. https://doi.org/10.3324/haematol.2013.087452

39. Chen X, Cheung ST, So S et al (2002) Gene expression patterns in human liver cancers. Mol Biol Cell 13:1929-1939. https:// doi.org/10.1091/mbc.02-02-0023

40. Li G-Q, Li H, Zhang H-F (2003) Mad2 and p53 expression profiles in colorectal cancer and its clinical significance. World J Gastroenterol 9:1972-1975

41. Sotillo R, Hernando E, Díaz-Rodríguez E et al (2007) Mad2 overexpression promotes aneuploidy and tumorigenesis in mice. Cancer Cell 11:9-23. https://doi.org/10.1016/j.ccr.2006.10.019

42. Boyapati A, Yan M, Peterson LF et al (2007) Aleukemia fusion protein attenuates the spindle checkpoint and promotes aneuploidy. Blood 109:3963-3971. https://doi.org/10.1182/blood -2006-09-045583

43. Lucena-Araujo AR, de Oliveira FM, Leite-Cueva SD et al (2011) High expression of AURKA and AURKB is associated with unfavorable cytogenetic abnormalities and high white blood cell count in patients with acute myeloid leukemia. Leuk Res 35:260-264. https://doi.org/10.1016/j.leukres.2010.07.034

44. Ye D, Garcia-Manero G, Kantarjian HM et al (2009) Analysis of Aurora kinase A expression in $\mathrm{CD}_{3}{ }^{+}$blast cells isolated from patients with myelodysplastic syndromes and acute myeloid leukemia. J Hematop 2:2-8. https://doi.org/10.1007/s1230 8-008-0019-3

45. Genga KR, Filho FDR, de Ferreira FVA et al (2015) Proteins of the mitotic checkpoint and spindle are related to chromosomal instability and unfavourable prognosis in patients with myelodysplastic syndrome. J Clin Pathol 68:381-387. https:// doi.org/10.1136/jclinpath-2014-202728

46. Will B, Zhou L, Vogler TO et al (2012) Stem and progenitor cells in myelodysplastic syndromes show aberrant stage-specific expansion and harbor genetic and epigenetic alterations. Blood. https://doi.org/10.1182/blood-2011-12-399683
47. Ricke RM, Jeganathan KB, van Deursen JM (2011) Bub1 overexpression induces aneuploidy and tumor formation through Aurora B kinase hyperactivation. J Cell Biol 193:1049-1064. https://doi.org/10.1083/jcb.201012035

48. Lin S-F, Lin P-M, Yang M-C et al (2002) Expression of hBUB1 in acute myeloid leukemia. Leuk Lymphoma 43:385-391. https ://doi.org/10.1080/10428190290006206

49. Ru HY, Chen RL, Lu WC, Chen JH (2002) hBUB1 defects in leukemia and lymphoma cells. Oncogene 21:4673-4679. https:// doi.org/10.1038/sj.onc. 1205585

50. Krapf G, Kaindl U, Kilbey A et al (2010) ETV6/RUNX1 abrogates mitotic checkpoint function and targets its key player MAD2L1. Oncogene 29:3307-3312. https://doi.org/10.1038/onc.2010.53

51. Foijer F, Xie SZ, Simon JE et al (2014) Chromosome instability induced by Mps1 and p53 mutation generates aggressive lymphomas exhibiting aneuploidy-induced stress. Proc Natl Acad Sci 111:13427-13432. https://doi.org/10.1073/pnas.1400892111

52. Döhner H, Estey E, Grimwade D et al (2017) Diagnosis and management of AML in adults: 2017 ELN recommendations from an international expert panel. Blood 129:424-447

53. Riegler J, Ebert A, Qin X et al (2016) Comparison of magnetic resonance imaging and serum biomarkers for detection of human pluripotent stem cell-derived teratomas. Stem Cell Rep 6:176187. https://doi.org/10.1016/j.stemcr.2015.12.008

54. Ohmine S, Salisbury JL, Ingle J et al (2018) Aurora-A overexpression is linked to development of aggressive teratomas derived from human iPS cells. Oncol Rep 39:1725-1730. https://doi. org/10.3892/or.2018.6239

55. Damelin M, Bestor TH (2007) The decatenation checkpoint. Br J Cancer 96:201-205. https://doi.org/10.1038/sj.bjc.6603537

56. Deming PB, Cistulli CA, Zhao H et al (2001) The human decatenation checkpoint. Proc Natl Acad Sci 98:12044-12049. https:// doi.org/10.1073/pnas.221430898

57. Luo K, Yuan J, Chen J, Lou Z (2009) Topoisomerase II?? Controls the decatenation checkpoint. Nat Cell Biol 11:204-210. https:// doi.org/10.1038/ncb1828

58. Deming PB, Flores KG, Stephen Downes C et al (2002) ATR enforces the topoisomerase II-dependent $\mathrm{G} 2$ checkpoint through inhibition of Plk1 kinase. J Biol Chem. https://doi.org/10.1074/ jbc.M206109200

59. Damelin M, Sun YE, Sodja VB, Bestor TH (2005) Decatenation checkpoint deficiency in stem and progenitor cells. Cancer Cell 8:479-484. https://doi.org/10.1016/j.ccr.2005.11.004

60. Greer Card DA, Sierant ML, Davey S (2010) Rad9A is required for G2 decatenation checkpoint and to prevent endoreduplication in response to topoisomerase II inhibition. J Biol Chem 285:15653-15661. https://doi.org/10.1074/jbc.M109.096156

61. Doherty SC, McKeown SR, McKelvey-Martin V et al (2003) Cell cycle checkpoint function in bladder cancer. J Natl Cancer Inst 95:1859-1868. https://doi.org/10.1093/jnci/djg120

62. Nakagawa T, Hayashita Y, Maeno K et al (2004) Identification of decatenation $\mathrm{G} 2$ checkpoint impairment independently of DNA damage G2 checkpoint in human lung cancer cell lines. Cancer Res 64:4826-4832. https://doi.org/10.1158/0008-5472. CAN-04-0871

63. Damelin M, Bestor TH (2006) Decatenation checkpoint deficiency destabilizes the stem cell genome. Cell Cycle 5:345-346. https:// doi.org/10.4161/cc.5.4.2480

64. Wray J, Williamson EA, Sheema S et al (2009) Metnase mediates chromosome decatenation in acute leukemia cells. Blood 114:1852-1858. https://doi.org/10.1182/blood-2008-08-175760

65. Lee S-H, Oshige M, Durant ST et al (2005) The SET domain protein Metnase mediates foreign DNA integration and links integration to nonhomologous end-joining repair. Proc Natl Acad Sci USA 102:18075-18080. https://doi.org/10.1073/pnas.0503676102 
66. Brooks K, Chia KM, Spoerri L et al (2014) Defective decatenation checkpoint function is a common feature of melanoma. J Invest Dermatol 134:150-158. https://doi.org/10.1038/jid.2013.264

67. Jain CK, Roychoudhury S, Majumder HK (2015) Selective killing of $\mathrm{G} 2$ decatenation checkpoint defective colon cancer cells by catalytic topoisomerase II inhibitor. Biochim Biophys Acta Mol Cell Res 1853:1195-1204. https://doi.org/10.1016/j.bbamc r.2015.02.021

68. Casenghi M, Mangiacasale R, Tuynder M et al (1999) p53-independent apoptosis and p53-dependent block of DNA rereplication following mitotic spindle inhibition in human cells. Exp Cell Res 250:339-350. https://doi.org/10.1006/excr.1999.4554

69. Mikule K, Delaval B, Kaldis P et al (2007) Loss of centrosome integrity induces p38-p53-p21-dependent G1-S arrest. Nat Cell Biol 9:160-170. https://doi.org/10.1038/ncb1529

70. Hanahan D, Weinberg RA (2000) The hallmarks of cancer. Cell 100:57-70. https://doi.org/10.1007/s00262-010-0968-0

71. Loeb KR, Loeb LA (2000) Significance of multiple mutations in cancer. Carcinogenesis 21:379-385

72. Rozhok AI, Salstrom JL, DeGregori J (2014) Stochastic modeling indicates that aging and somatic evolution in the hematopoietic system are driven by non-cell-autonomous processes. Aging (Albany NY) 6:1033-1048. https://doi.org/10.18632/aging.10070 7

73. Lorenzini A, Fink LS, Stamato T et al (2011) Relationship of spindle assembly checkpoint fidelity to species body mass, lifespan, and developmental rate. Aging (Albany NY) 3:1206-1212. https ://doi.org/10.18632/aging.100416

74. Kung AL, Sherwood SW, Schimke RT (1990) Cell line-specific differences in the control of cell cycle progression in the absence of mitosis. Proc Natl Acad Sci USA 87:9553-9557. https://doi. org/10.1073/pnas.87.24.9553

75. Duncan FE, Chiang T, Schultz RM, Lampson MA (2009) Evidence that a defective spindle assembly checkpoint is not the primary cause of maternal age-associated aneuploidy in mouse eggs. Biol Reprod 81:768-776. https://doi.org/10.1095/biolreprod .109 .077909
76. Baker DJ, Jeganathan KB, Cameron JD et al (2004) BubR1 insufficiency causes early onset of aging-associated phenotypes and infertility in mice. Nat Genet 36:744-749. https://doi.org/10.1038/ ng 1382

77. Tomás-Loba A, Flores I, Fernández-Marcos PJ et al (2008) Telomerase reverse transcriptase delays aging in cancer-resistant mice. Cell 135:609-622. https://doi.org/10.1016/j.cell.2008.09.034

78. Ikezoe T, Yang J, Nishioka C et al (2007) A novel treatment strategy targeting Aurora kinases in acute myelogenous leukemia. Mol Cancer Ther 6:1851-1857. https://doi.org/10.1158/1535-7163. MCT-07-0067

79. Bavetsias V, Linardopoulos S (2015) Aurora kinase inhibitors: current status and outlook. Front Oncol. https://doi.org/10.3389/ fonc. 2015.00278

80. Kiyomitsu T, Murakami H, Yanagida M (2011) Protein interaction domain mapping of human kinetochore protein Blinkin reveals a consensus motif for binding of spindle assembly checkpoint proteins Bub1 and BubR1. Mol Cell Biol 31:998-1011. https:// doi.org/10.1128/MCB.00815-10

81. Renner AG, Dos Santos C, Recher C et al (2009) Polo-like kinase 1 is overexpressed in acute myeloid leukemia and its inhibition preferentially targets the proliferation of leukemic cells. Blood 114:659-662. https://doi.org/10.1182/blood-2008-12-195867

82. Yoshida K, Miki Y (2004) Role of BRCA1 and BRCA2 as regulators of DNA repair, transcription, and cell cycle in response to DNA damage. Cancer Sci 95:866-871

83. Morgado-Palacin I, Day A, Murga M et al (2016) Targeting the kinase activities of ATR and ATM exhibits antitumoral activity in mouse models of MLL-rearranged AML. Sci Signal 9:ra91. https ://doi.org/10.1126/scisignal.aad8243

84. Sallmyr A, Tomkinson AE, Rassool FV (2008) Up-regulation of WRN and DNA ligase IIIalpha in chronic myeloid leukemia: consequences for the repair of DNA double-strand breaks. Blood 112:1413-1423. https://doi.org/10.1182/blood-2007-07-104257 